\title{
Microscopic recoil model for light-cone fluctuations in quantum gravity
}

\author{
John Ellis \\ Theory Division, CERN, CH-1211 Geneva 23, Switzerland \\ N. E. Mavromatos \\ Department of Physics, University of Oxford, 1 Keble Road, Oxford OX1 3NP, United Kingdom \\ and Theory Division, CERN, CH-1211 Geneva 23, Switzerland \\ D. V. Nanopoulos \\ Department of Physics, Texas A \& M University, College Station, Texas 77843-4242; \\ Astroparticle Physics Group, Houston Advanced Research Center (HARC), Mitchell Campus, Woodlands, Texas 77381; \\ and Academy of Athens, Chair of Theoretical Physics, Division of Natural Sciences, 28 Panepistimiou Avenue, Athens 10679, Greece
}

(Received 11 June 1999; published 23 December 1999)

\begin{abstract}
We present a microscopic model for light-cone fluctuations in vacuo, which incorporates a treatment of quantum-gravitational recoil effects induced by energetic particles. Treating defects in space-time as solitons in string theory, we derive an energy-dependent refractive index and a stochastic spread in the arrival times of mono-energetic photons due to quantum diffusion through space-time foam, as found previously using an effective Born-Infeld action. Distant astrophysical sources provide sensitive tests of these possible quantumgravitational phenomena.
\end{abstract}

PACS number(s): 04.60. $-\mathrm{m}, 04.62 .+\mathrm{v}, 11.25 .-\mathrm{w}$

The theory of quantum gravity is still elusive, despite the progress made in recent years in various different approaches, including those based on canonical field theory [1] and on the theory formerly known as string theory [2]. Quantum fluctuations in the underlying space-time, such as might lead to the formation and evaporation of microscopic black holes, are still not understood, although the modern representation of defects in space-time as string solitons has yielded deep insight into massive black holes in flat space-time.

Quantum-gravitational fluctuations in the space-time background might cause the vacuum to have non-trivial optical properties, due in particular to recoil induced by a propagating energetic particle. An important issue in the study of such effects is whether manifest Lorentz invariance is retained. In our view $[3,4]$, as well as that of others $[1,5-$ 7], a quantum-gravity ground state may not be Lorentz invariant since the quantization of the metric field about a given background may induce a preferred frame. This could be regarded as a spontaneous breaking of Lorentz invariance, which is probably a necessary step in viewing the gravitational vacuum as a "medium." Such a symmetry breakdown is inevitable in the canonical approach to quantizing the gravitational field, which leads to quantum fluctuations in the light-cone. We and others have suggested that the breakdown of Lorentz invariance may lead to observable consequences for the propagation of light [3-5], and the main purpose of this Brief Report is to derive these via a microscopic recoil model for light-cone fluctuations.

Light propagating through media with non-trivial optical properties may exhibit a frequency-dependent refractive index, namely a variation in the light velocity with photon energy. Another possibility is a difference between the velocities of light with different polarizations, namely birefringence, and a third is a diffusive spread in the apparent velocity of light for light of fixed energy (frequency). We have derived the first [3] and third [4] effects previously using a formal approach based on a Born-Infeld Lagrangian obtained from solitonic effects in string theory, and the possibility of birefringence has been raised [5] within a canonical approach to quantum gravity. A different approach to light propagation has been taken in [6], where quantum-gravitational fluctuations in the light-cone have been calculated. In this paper, we use this formalism together with a microscopic model background obtained from considering the quantum recoil of a string soliton to derive a non-trivial refractive index and a diffusive spread in the arrival times of photons of given frequency.

We first review briefly the analysis in [6], which considered gravitons in a squeezed coherent state, the natural result of quantum creation in the presence of black holes. Such gravitons induce quantum fluctuations in the spacetime metric, in particular fluctuations in the light-cone [6], i.e., stochastic fluctuations in the velocity of light propagating through this "medium," which may have observable effects $\Delta t$ on the arrival times of photons. Following [6], we consider a flat background space-time with a linearized perturbation, corresponding to the invariant metric element $d s^{2}=g_{\mu \nu} d x^{\mu} d x^{\nu}=\left(\eta_{\mu \nu}+h_{\mu \nu}\right) d x^{\mu} d x^{\nu}=d t^{2}-d \bar{x}^{2}$ $+h_{\mu \nu} d x^{\mu} d x^{\nu}$. Let $2 \sigma\left(x, x^{\prime}\right)$ be the squared geodesic separation for any pair of space-time points $x$ and $x^{\prime}$, and let $2 \sigma_{0}\left(x, x^{\prime}\right)$ denote the corresponding quantity in a flat spacetime background. In the case of small gravitational perturbations about the flat background, one may expand $\sigma=\sigma_{0}$ $+\sigma_{1}+\sigma_{2}+\cdots$, where $\sigma_{n}$ denotes the $n$-th order term in an expansion in the gravitational perturbation $h_{\mu \nu}$. Then, as shown in [6], the root-mean-square (RMS) deviation from the classical propagation time $\Delta t$ is related to $\left\langle\sigma^{2}\right\rangle$ by

$$
\Delta t=\frac{\sqrt{\left\langle\sigma^{2}\right\rangle-\left\langle\sigma_{0}^{2}\right\rangle}}{L} \simeq \frac{\sqrt{\left\langle\sigma_{1}^{2}\right\rangle}}{L}+\cdots
$$


where $L=\left|x^{\prime}-x\right|$ is the distance between the source and the detector. The expression (1) is gauge invariant [6].

The model we propose in this paper is based on solitons in string theory, which are described formally as $D$ branes [2]. As commented earlier, one may expect Lorentz invariance to be broken in a generic theory of quantum gravity. In the context of string theory, this entails the exploration of non-critical string backgrounds, since Lorentz invariance is related to the conformal symmetry that is a property of critical string theory. A general approach to the formulation of non-critical string theory involves introducing a Liouville field [8] as a conformal factor on the string world sheet, which has non-trivial dynamics and compensates the nonconformal behavior of the string background. In the case of $D$-brane string solitons, their recoil after interaction with a closed-string state [9], as used to describe a photon, is characterized by a pair of logarithmic operators [10]:

$$
C_{\epsilon} \sim \epsilon \Theta_{\epsilon}(t), \quad D_{\epsilon} \sim t \Theta_{\epsilon}(t)
$$

defined on the boundary $\partial \Sigma$ of the string world sheet.

The operators (2) act as deformations of the conformal field theory on the world sheet: $U_{i} \int_{\partial \Sigma} \partial_{n} X^{i} D_{\epsilon}$ describes the shift of the $D$ brane induced by the scattering, where $U_{i}$ is its recoil velocity, and $Y_{i} \int_{\partial \Sigma} \partial_{n} X^{i} C_{\epsilon}$ describes quantum fluctuations in the initial position $Y_{i}$ of the $D$ particle. It has been shown [11] that energy-momentum is conserved during the recoil process: $U_{i}=k_{1}-k_{2}$, where $k_{1}\left(k_{2}\right)$ is the momentum of the propagating closed-string state before (after) the recoil, as a result of the summation over world-sheet genera. We also note that $U_{i}=g_{s} P_{i}$, where $P_{i}$ is the momentum and $g_{s}$ is the string coupling, which is assumed here to be weak enough to ensure that $D$ branes are very massive, with mass $M_{D}=1 /\left(l_{s} g_{s}\right)$, where $l_{s}$ is the string length.

The correct specification of the logarithmic pair (2) entails a regulating parameter $\epsilon \rightarrow 0^{+}$, which appears inside the $\Theta(t)$ operator: $\Theta(t)=\int(d \omega / 2 \pi)[1 /(\omega-i \epsilon)] e^{i \omega t}$. In order to realize the logarithmic algebra between the operators $C$ and $D$, one takes [9]: $\epsilon^{-2} \sim \log \Lambda / a \equiv \alpha$, where $\Lambda$ (a) are infrared (ultraviolet) world-sheet cutoffs. The recoil operators (2) are slightly relevant, in the sense of the renormalization group for the world-sheet field theory, with small conformal dimensions $\Delta_{\epsilon}=-\epsilon^{2} / 2$. The relevant two-point functions have the following form:

$$
\begin{gathered}
\left\langle C_{\epsilon}(z) C_{\epsilon}(0)\right\rangle \stackrel{\epsilon \rightarrow 0}{\sim} 0+\mathcal{O}\left(\epsilon^{2}\right) \\
\left\langle C_{\epsilon}(z) D_{\epsilon}(0)\right\rangle \stackrel{\epsilon \rightarrow 0}{\sim} \frac{\pi}{2} \sqrt{\frac{\pi}{\epsilon^{2} \alpha}}\left(1-\left.2 \eta \epsilon^{2} \log |z| a\right|^{2}\right) \\
\left\langle D_{\epsilon}(z) D_{\epsilon}(0)\right\rangle=\frac{1}{\epsilon^{2}}\left\langle C_{\epsilon}(z) D_{\epsilon}(0)\right\rangle \\
\epsilon \rightarrow 0 \\
\sim \frac{\pi}{2} \sqrt{\frac{\pi}{\epsilon^{2} \alpha}}\left(\frac{1}{\epsilon^{2}}-2 \eta \log |z / a|^{2}\right)
\end{gathered}
$$

which is the logarithmic algebra [10] in the limit $\epsilon \rightarrow 0^{+}$, modulo the leading divergence in the $\left\langle D_{\epsilon} D_{\epsilon}\right\rangle$ recoil cor- relator. In fact, it is this leading divergent term that will be of importance for our purposes in this article.

It is the fact that the recoil operators are relevant operators in a world-sheet renormalization-group sense that requires dressing the world-sheet theory with a Liouville field [8] in order to restore conformal invariance, which has been lost in the recoil process. One then makes the crucial step of identifying the world-sheet zero mode of the Liouville field with the target time $t$, which is justified in $[12,11]$ using the logarithmic algebra (3) for the case at hand. This identification leads to the appearance of a curved space-time background in target space, with the metric elements [13]

$$
G_{i j}=\delta_{i j}, \quad G_{00}=-1, \quad G_{0 i}=\epsilon\left(\epsilon Y_{i}+U_{i} t\right) \Theta_{\epsilon}(t)
$$

where the suffix 0 denotes temporal (Liouville) components.

We now remark [11] that the velocity operator $D_{\epsilon}$ (2) becomes exactly marginal, in a world-sheet renormalizationgroup sense, upon division by $\epsilon$, in which case the recoil velocity is renormalized: $U_{i} \rightarrow \bar{U}_{i} \equiv U_{i} / \epsilon$ is the physical recoil velocity. Viewed as a perturbation about a flat target space-time, the metric (4) implies that the only non-zero components of $h_{\mu \nu}$ are

$$
h_{0 i}=\epsilon^{2} \bar{U}_{i} t \Theta_{\epsilon}(t)
$$

in the case of $D$-brane recoil.

Consider light propagation along the $x$ direction in the presence of a metric fluctuations $h_{0 x}$ (5) in flat space, along a null geodesic given by $(d t)^{2}=(d x)^{2}+2 h_{0 x} d t d x$. For large times $t \sim \log \Lambda / a \sim \epsilon^{-2}[13], h_{o x} \sim \bar{U}$, and thus we obtain

$$
\frac{c d t}{d x}=\bar{U}+\sqrt{1+\bar{U}^{2}} \sim 1+\bar{U}+\mathcal{O}\left(\bar{U}^{2}\right)
$$

where the recoil velocity $\bar{U}$ is in the direction of the incoming light ray. Taking into account energy-momentum conservation in the recoil process, which has been derived in this formalism as mentioned previously, one has a typical order of magnitude $\bar{U} / c=\mathcal{O}\left(E / M_{D} c^{2}\right)$, where $M_{D}=g_{s}^{-1} M_{s}$ is the $D$-brane mass scale, with $M_{s} \equiv l_{s}^{-1}$. Hence Eq. (6) implies a subluminal energy-dependent velocity of light:

$$
c(E) / c=1-\mathcal{O}\left(E / M_{D} c^{2}\right)
$$

which corresponds to a classical refractive index. This appears because the metric perturbation (5) is energydependent, through its dependence on $\bar{U}$.

The subluminal velocity (7) induces a delay in the arrival of a photon of energy $E$ propagating over a distance $L$ of order:

$$
(\Delta t)_{r}=\frac{L}{c} \mathcal{O}\left(\frac{E}{M_{D} c^{2}}\right) .
$$

This effect can be understood physically from the fact that the curvature of space-time induced by the recoil is $\bar{U}$ - and hence energy-dependent. This affects the paths of photons in 
such a way that more energetic photons see more curvature, and thus are delayed with respect to low-energy ones.

The absence of superluminal light propagation was found previously via the formalism of the Born-Infeld Lagrangian dynamics of $D$ branes $[11,4]$. Furthermore, the result (8) is in agreement with the analysis of [14,3], which was based on a more abstract analysis of Liouville strings. It is encouraging that this follows also from a more conventional general relativity approach [6], in which the underlying physics is transparent. We find no birefringence effect, in contrast to [5].

As a preliminary to evaluating quantum effects [4] in the context of our simplified one-dimensional problem, we now express $\sigma_{1}^{2}$ in terms of the two-point function of $h_{0 x}$, considering again the null geodesic in the presence of the small metric perturbations (5). To first order in the recoil velocity, one has $c(\Delta t)=\left(\Delta x-2 \int_{x_{1}}^{x_{2}} d x h_{0 x}\right)$, from which we find $2 \sigma \simeq(\Delta t)^{2}-(\Delta x)^{2}+2 \epsilon^{2} \bar{U} t \Theta_{\epsilon}(t)(\Delta x)^{2}$, implying that $\sigma_{1}=\epsilon^{2} \bar{U} t \Theta_{\epsilon}(t)$. One then has

$$
\left\langle\sigma_{1}^{2}\right\rangle \sim L^{2} \int_{x}^{x^{\prime}} \int_{x}^{x^{\prime}} d y \int d y^{\prime}\left\langle h_{0 x}(y, t) h_{0 x}\left(y^{\prime}, t^{\prime}\right)\right\rangle .
$$

In the case of $D$-brane recoil, the computation of the quantum average $\langle\cdots\rangle$ may be made in the Liouville-string theory approach described above. In this case, the quantum average $\langle\cdots\rangle$ is replaced by a world-sheet correlator calculated with a world-sheet action deformed by Eq. (2). It is clear from Eq. (5) that the two-point metric correlator appearing in Eq. (9) is just the $\left\langle D_{\epsilon} D_{\epsilon}\right\rangle$ world-sheet recoil twopoint function described in Eq. (3). Thus, at the classical tree level on the world sheet, one may recover the refractive index (8) from Eq. (9) by concentrating on the leading divergence in the $D_{\epsilon} D_{\epsilon}$ correlator (3), proportional to $\epsilon^{-2}$.

To describe fully the quantum effects, one must sum over world-sheet genera. As discussed in $[12,11]$ such a procedure results in a canonical quantization of the world-sheet couplings, which, in the problem at hand, coincide with the target-space collective coordinates and momenta of the recoiling $D$ brane. Thus, the quantum effects arising from summation over world-sheet genera result, in a first approximation, in $\left\langle\sigma_{1}^{2}\right\rangle \sim L^{4}(\Delta \bar{U})^{2}$, where $\Delta \bar{U}$ denotes the quantum uncertainty of the recoil velocity $\bar{U}$, which has been computed in [11].

The result of the summation over genera has been discussed in detail in $[12,11]$, and is not repeated here: we only state the final results relevant for our purposes. The leading contributions to the quantum fluctuations in the space $\mathcal{M}$ of world-sheet couplings arise from pinched annulus diagrams in the summation over world-sheet general $[12,11]$. These consist of thin tubes of width $\delta \rightarrow 0$, which one may regard as wormholes attached to the world-sheet surface $\Sigma$. The attachment of each tube corresponds to the insertion of a bilocal pair of recoil vertex operators $V^{i}(s) V^{j}\left(s^{\prime}\right)$ on the boundary $\partial \Sigma$, with interaction strength $g_{s}^{2}$, and computing the string propagator along the thin tubes. One effect of the dilute gas of world-sheet wormholes is to exponentiate the bilocal operator, leading to a change in the world-sheet ac- tion $[12,11]$. This contribution can be cast into the form of a local action by rewriting it as a Gaussian functional integral over wormhole parameters $\rho_{i}^{a b}$, leading finally to [11]

$$
\sum_{\text {genera }} Z \simeq \int_{\mathcal{M}} D \rho e^{-\rho_{i}^{a b} G_{a b, c d}^{i j} \rho_{j}^{c d} / 2|\epsilon|^{2} l_{s}^{2} g_{s}^{2} \log \delta}\langle W[Y+\rho]\rangle_{0}
$$

where $\langle W[Y+\rho]\rangle_{0}$ denotes the partition function on a world sheet with the topology of a disc of a model deformed by the operators (2), with couplings shifted by $\rho$, and $G^{i j}$ is the inverse of the Zamolodchikov metric [15] $\left\langle V_{i} V_{j}\right\rangle$ in string theory space, evaluated on the disc world sheet. The shifts in the effective couplings $Y_{i}, U_{i}$ imply that they fluctuate statistically, in much the same way as wormholes and other topology changes in target space times lead to the quantization of the couplings in conventional field theories [16].

In our case, we see from Eq. (10) that the effect of this resummation over pinched genera is to induce quantum fluctuations in the solitonic $D$-brane background, giving rise to a Gaussian statistical spread in the collective coordinates of the $D$ brane, determined by $G^{i j}$ for the logarithmic deformations (2). Note that, in such a formalism, one defines a renormalized string coupling $\bar{g}_{s}=g_{s} \epsilon^{-1}$, which plays the role of the physical string coupling in the problem [11]. To lowest non-trivial order in the string coupling $\bar{g}_{s}$ and $\bar{U}^{2}$, the worldsheet analysis of [11], using the methods of logarithmic conformal field theory [10], showed that the quantum fluctuations in $\bar{U}$ induced by the summation over world-sheet topologies are given by

$$
(\Delta \bar{U})^{2}=4 \bar{g}_{s}^{2}\left[1-\frac{285}{2} \bar{U}^{2}\right]
$$

if the $D$-brane foam corresponds to a minimum-uncertainty wave-packet [11], with the position fluctuations of the $D$ branes being saturated: $\Delta Y_{i} \sim \bar{g}_{s}^{-1 / 3} l_{s}$. The energyindependent first part of Eq. (11), can be absorbed into $\sigma_{0}$, and hence does not contribute to the stochastic fluctuations (1) in the photon arrival time. The second part of the uncertainty (11), which depends on $\bar{U}$, and hence the energy of the photon, cannot be scaled away for all photons of different energies by a simple coordinate transformation. However, it is of higher (second) order in the small parameter $\bar{U}$. Thus the geodesic correction $\sigma_{1}$, which is linear in the gravitational perturbation $h_{\mu \nu}$, leads in leading order only to a classical refractive index.

We now repeat the analysis for the quadratic correction $\sigma_{2}$ in Eq. (1). This contains the normal-ordered coincidence limit of the two-point function of the $D_{\epsilon}$ operator, which has a leading-order term proportional to $\epsilon^{-2}$ times the $c$-number identity, as seen in Eq. (3). Since we work in a subtraction scheme in which one-point functions of the deformations (2) vanish, this means that $\left\langle\sigma_{1} \sigma_{2}\right\rangle=0$, so that the leading contribution comes from the sum over genera of $\left\langle\sigma_{2}^{2}\right\rangle$, which yields fluctuations in the arrival time that are proportional to the uncertainty $\Delta\left(\bar{U}^{2}\right) \sim \bar{U} \Delta \bar{U}$. For a minimum-uncertainty state of $D$ branes, we therefore find a contribution 


$$
\left|(\Delta t)_{o b s}\right| \simeq \mathcal{O}\left(\bar{g}_{s} \frac{E}{M_{D} c^{2}}\right) \frac{L}{c}
$$

to the RMS fluctuation in arrival times. As expected, the quantum effect (12) is suppressed by a power of the string coupling constant, when compared with the classical refractive index effect (8). The result (12) was derived in [4] using the techniques of Liouville string theory, via the Born-Infeld Lagrangian for the propagation of photons in the $D$-brane foam. Its derivation here using more conventional fieldtheoretic techniques adds robustness to the Liouville string theory perspective. It should be noted that the recoil-induced effect (12) is larger than the effects discussed in [6], which are related to metric perturbations associated with the squeezed coherent states relevant to particle creation in conventional local field theories. The recoil effects discussed here are also related to particle creation, but in a string soliton context [13].

We close by recalling that the energy-dependent departures from an absolute velocity of light that have been discussed here are amenable to experimental probes. The most promising may involve observations of distant astrophysical objects whose emissions exhibit structures within short times $\delta t$ [3], such as gamma-ray bursters (GRBs), pulsars and active galactic nuclei (AGNs), with a premium on measurements of the arrival times of high-energy photons, so as to maximize the figure of merit $M_{Q G} \equiv L \times E / \delta t$. Past and present observations of such sources have all been shown to be sensitive to $M_{Q G} \sim 10^{16} \mathrm{GeV}$ [17], within a few orders of magnitude of the Planck mass $M_{P} \sim 10^{19} \mathrm{GeV}$. We may hope that future generations of $\gamma$-ray telescopes with larger collection areas (so as to extend the reach in energy E) and accurate timing (so as to minimize $\delta t$ ), such as AMS, GLAST and VERITAS, will be able to benefit from future identifications of high-redshift sources (so as to maximize $L$ ), pushing the experimental sensitivity into the uncharted territory of quantum gravity.

The work of N.E.M. is supported in part by P.P.A.R.C. and that of D.V.N. by D.O.E. grant DE-FG03-95-ER-40917. We gratefully acknowledge the support of Hans Hofer and the interest of Marta Felcini.
[1] For a recent review, see A. Ashtekar, gr-qc/9901023.

[2] For a recent review, see A. Sen, "Lectures given at Cambridge University, U.K.,' hep-th/9802051.

[3] G. Amelino-Camelia, J. Ellis, N.E. Mavromatos, D.V. Nanopoulos, and S. Sarkar, Nature (London) 393, 323 (1998).

[4] J. Ellis, N.E. Mavromatos, and D.V. Nanopoulos, Gen. Relativ. Gravit. 31, 1257 (1999); Gen. Relativ. Gravit. (to be published), gr-qc/9904068.

[5] R. Gambini and J. Pullin, Phys. Rev. D 59, 124021 (1999).

[6] L.H. Ford, Phys. Rev. D 51, 1692 (1995); H. Yu and L.H. Ford, ibid. 60, 084023 (1999).

[7] Luis J. Garay, Phys. Rev. Lett. 80, 2508 (1998); Phys. Rev. D 58, 124015 (1998).

[8] F. David, Mod. Phys. Lett. A 3, 1651 (1988); J. Distler and H. Kawai, Nucl. Phys. B321, 509 (1989).

[9] I.I. Kogan, N.E. Mavromatos, and J.F. Wheater, Phys. Lett. B 387, 483 (1996).

[10] V. Gurarie, Nucl. Phys. B410, 535 (1993).
[11] N.E. Mavromatos and R.J. Szabo, Phys. Rev. D 59, 104018 (1999); F. Lizzi and N.E. Mavromatos, ibid. 55, 7859 (1997).

[12] J. Ellis, N.E. Mavromatos, and D.V. Nanopoulos, Int. J. Mod. Phys. A 12, 2639 (1997); 13, 1059 (1998).

[13] J. Ellis, P. Kanti, N.E. Mavromatos, D.V. Nanopoulos, and E. Winstanley, Mod. Phys. Lett. A 13, 303 (1998).

[14] G. Amelino-Camelia, J. Ellis, N.E. Mavromatos, and D.V. Nanopoulos, Int. J. Mod. Phys. A 12, 607 (1997).

[15] A.B. Zamolodchikov, Pis'ma Zh. Éksp. Teor. Fiz. 43, 565 (1986) [JETP Lett. 43, 730 (1986)]; Yad. Fiz. 46, 1819 (1987) [Sov. J. Nucl. Phys. 46, 1090 (1987)].

[16] S. Coleman, Nucl. Phys. B310, 643 (1988).

[17] S.D. Biller et al., Phys. Rev. Lett. 83, 2108 (1999); P. Kaaret, Astron. Astrophys. 345, 32 (1999); astro-ph/9903464; S. Ahlen et al., Nucl. Instrum. Methods Phys. Res. A 350, 351 (1994); GLAST Team, E.D. Bloom et al., in Proceedings of the International Heidelberg Workshop on TeV Gamma-Ray Astrophysics, edited by H.J. Volk and F.A. Aharonian (Kluwer, Dordrecht, 1996), p. 109. 